\title{
On Stable Reconstruction of the Impact in the System of Ordinary Differential Equations
}

\author{
Andrei Y. Vdovin, Svetlana S. Rubleva \\ The Ural State Forest-Engineering University, Ekaterinburg, Russia \\ E-mail: rublevas@mail.ru \\ Received February 18, 2010; revised May 27, 2010; accepted June 8, 2010
}

\begin{abstract}
Approach to expansion of an opportunity of the reception the guaranteed estimation for a problem of reconstruction the impact within the limits of the dynamical algorithm is considered in the article.
\end{abstract}

Keywords: Dynamical Algorithm, The Reconstruction of the Impact, The Estimation of Accuracy of the Algorithm

\section{Problem Statement}

Consider the problem of reconstruction of entrance impact $u(\cdot)$ in the dynamical system

$$
x^{\prime}(t)=p(t, x(t))+f(t, x(t)) u(t), x(a)=x_{a}, t \in[a, b],
$$

according to inexact measurements $x_{h}(\cdot)$ of states $x(\cdot)$ of the system (1) in knots of splitting [a,b]: $a=t_{0}<t_{1}$ $<\ldots<t_{n}=b:\left|x_{h}\left(t_{i}\right)-x\left(t_{i}\right)\right| \leqslant h$.

Here $p(\cdot), f(\cdot)$ are mappings from $[\mathrm{a}, \mathrm{b}] \times R^{m}$ into $R^{m}$ (with Euclidean norm $|\cdot|$ ) and into $R^{m \times q}$ - the matrix space of dimension $m \times q$ (with spectral norm $\|\cdot\|$ ), respectively; values of the impact $u(\cdot)$ belong to compactum $Q \subset R^{q}$, and values of the $x(t)$ belong to compactum $X \subset R^{m}$.

The problem in such statement has been widely covered in the literature. For its decision we will adhere to the approach, considered in [1]. It was offered to restore the impact $u_{*}(\cdot)$ with the minimum norm in $L_{2}$ [a,b] among all impacts $u(\cdot)$ generating observable movement $x(\cdot)$ for stability of algorithm.

Essence of method consists in the following: let $\alpha(\cdot)$, $\Delta(\cdot):(0, \infty) \rightarrow(0, \infty),\langle\cdot$,$\rangle - scalar product, comp-$ actum $Q$ is convex, and index $T$ is denote transposing.

In each partition interval $\left[t_{i}, t_{i+1}\right)$ are formed:

1 ) the value at the point $t_{i+1}$ of the system of the model functioning according to the rule

$$
w_{h}(t)=w_{h}\left(t_{i}\right)+\left(p\left(t_{i}, x_{h}\left(t_{i}\right)\right)+f\left(t_{i}, x_{h}\left(t_{i}\right)\right) u_{i}\right)\left(t-t_{i}\right),
$$

2) the value of $u_{i}$, being the result of projection on $Q$ of the vector

$$
\frac{1}{\alpha(h)} f^{T}\left(t_{i}, x_{h}\left(t_{i}\right)\right)\left(x_{h}\left(t_{i}\right)-w_{h}\left(t_{i}\right)\right) .
$$

So, the considered algorithm (further $D_{h}$ ) puts in conformity to measurement $x_{h}(\cdot)$ the piecewise constant approximation $u_{h}(\cdot)$ of the impact $u_{*}(\cdot)$, where $u_{h}(t)=u_{i}$, $t \in\left[t_{i}, t_{i+1}\right)$.

Suppose that $\rho_{F}(h)=\sup _{x_{h}(\cdot)}\left\|u_{h}(\cdot)-u_{*}(\cdot)\right\|_{F[a, b]}$, where $F[a, b]$ - some functional space. If $\lim _{h \rightarrow 0} \rho_{F}(h)=0$, then algorithm is called $F[a, b]$ - regularizing.

Statement 1: [1] Suppose that $x(t)$ at $t \in[a, b]$ belong to compactum $X$ from $R^{m}$; functions $p(t, x), f(t, x)$ at $(t, x(t)) \in[a, b] \times X$ are Lipschitz with respect to all the variables with common constant $L$; parameters $\alpha(h), \Delta(h)$ are vanishing together with $h$ so that $\lim _{h \rightarrow 0} \frac{h+\Delta(h)}{\alpha(h)}=0$. Then $D_{h}-L_{2}[\mathrm{a}, \mathrm{b}]$ - regularizing.

The question on estimations of accuracy of algorithm is essential at its use. Let's enter the following concepts:

Definition. A function $v_{1}(h)\left(v_{2}(h)\right):\left[0, h_{*}\right) \rightarrow[0, \infty)$ is called a lower (upper) estimate of the accuracy of the algorithm $D_{h}$ in a space $F[a, b]$, if for all $h \in\left(0, h_{*}\right]$ inequalities $v_{1}(h) \leqslant \rho_{F}(h) \leqslant v_{2}(h)$ hold, and $\gamma(h):[0$, $\left.h_{*}\right) \rightarrow[0, \infty)$ is called the order of the accuracy of the algorithm $D_{h}$ in $F[a, b]$, if there exist positive constant $C_{i}, i=1,2$ such that $C_{1} h^{\gamma(h)} \leqslant \rho_{F}(h) \leqslant C_{2} h^{\gamma(h)}$. 
The number $\gamma_{0}$ is called asymptotic order of accuracy of the algorithm $D_{h}$ in $F[a, b]$, if $\lim _{h \rightarrow 0} \gamma(h)=\gamma_{0}$ exists.

The estimations of the accuracy for the discontinuous impact in space $L_{1}[a, b]$ for the described algorithm are received, for example [2,3]. The purpose of the article is construction of modification $D_{h}$ [4] and indication the additional assumptions at which receipting of asymptotic order in $C[a, b]$ is possible. For this purpose we will adhere to the approach offered in [2], therefore we will omit common proves of lemmas.

Note, that on first step of work of the algorithm as approximation of $u_{*}(\cdot)$ we select projection of zero on $Q$. The last make receipt of estimation with condition $\lim _{h \rightarrow 0} v_{2}(h)=0$ is impossible. If the initial condition $u(a)$ is known (we fix left end of interval), then system (1) can be led to the kind:

$$
x^{\prime}(t)=g(t, x(t))+f(t, x(t)) v(t), x(a)=x_{a}, v(a)=0
$$

where $v(t)=u(t)-u(a), g(t, x(t))=p(t, x(t))+f(t, x(t)) u(a)$, $t \in[a, b]$.

Consider the problem of reconstruction of impact $v_{*}(\cdot)$ in new system (2).

In what follows, we assume of performance of the following set of conditions:

Condition (*). In additional to the assertions of statement 1 we suppose that for all $t \in[a, b] 1) \operatorname{rank}(f(t, x(t)))$ is equal $r, 2) v_{*}(\cdot)$ is satisfied to condition of Lipschitz with constant $\left.L_{v} ; 3\right)$ the infima with respect to t of distances between the boundaries of compactumes $Q$ and $X$ and $v_{*}(t)$ and $x(t)$, respectively, are positive; 4) the value $v(a)$ is known with some error: $\left|v(a)-v_{\sigma}(a)\right| \leqslant \sigma(h)$.

Remark. Condition (*) involves the existence of positive constants $M_{f}, M_{g}, M_{v}$ such that $\|f(\cdot)\| \leqslant M_{f},|g(\cdot)|$ $\leqslant M_{g},|v(\cdot)| \leqslant M_{v}$.

In the modification $D_{h}^{(1)}$ of the algorithm $D_{h}$ besides transformation of the kind of system we refuse from procedure of projection on compactum $Q$. The last decrease of arithmetical operations executed at each step, in which case the approximation for $v_{h}(\cdot)$, where $t \in\left[t_{i}, t_{i+1}\right)$, is given by the formula:

$$
v_{i}=f^{T}\left(t_{i}, x_{h}\left(t_{i}\right)\right) \frac{x_{h}\left(t_{i}\right)-w_{h}\left(t_{i}\right)}{\alpha(h)} .
$$

Let us fix $\alpha(h)$. The vector

$$
v_{0}(t)=\frac{1}{\alpha(h)} f^{T}(t, x(t))\left(x(t)-w_{0}(t)\right)
$$

and the system - model

$$
w_{0}^{\prime}(t)=g(t, x(t))+A(t, x(t)) \frac{x(t)-w_{0}(t)}{\alpha(h)}, w_{0}(a)=x_{a},
$$

where

$$
A(t, x(t))=f(t, x(t)) f^{T}(t, x(t))
$$

we'll name the ideal.

In practice, it is impossible to construct $v_{0}(\cdot)$ on the basis of measurement of $x_{h}(\cdot)$, but, in what follows, we shall only need estimates of $\left|v_{0}(t)-v_{*}(t)\right|$ and the norms of difference between $v_{0}(\cdot)$ and $v_{h}(\cdot)$, which allow us to obtain the asymptotic order of accuracy of $D_{h}^{(1)}$.

\section{Estimation of Norm of a Difference $v_{0}(\cdot)$ and $v *(\cdot)$}

Let's consider some important statements.

Statement 2: [5,6] If $H$ - arbitrary matrix, $H^{+}$is its pseudo-inverse, then equalities $H=H H^{T}\left(H^{T}\right)^{+}, H H^{+}=$ $\left(H H^{+}\right)^{T},\left(H^{T}\right)^{+}=\left(H^{+}\right)^{T}$ are valid.

Statement 3: [5,7] If $t \in[a, b]$, and the matrix $A(t$, $x(t))$ is defined by equality (4), then its eigenvalues $\lambda_{k}(t), k=\overline{1, m}$ are non-negatives: $\lambda_{1}(t) \geqslant \ldots \geqslant \lambda_{r}(t)>0$, $\lambda_{r+1}(t)=\ldots=\lambda_{m}(t)=0$, and $\left\|A^{+}(t, x(t))\right\| \leqslant \frac{1}{\lambda_{r}(t)}$.

Statement 4: [5] If matrix $H \in R^{m \times m}$ is hermitian, $R_{0}(H)$ is its kernel, and $R_{1}(H)$ its image, then $R^{m}=R_{0}(H)$ $\oplus R_{1}(H)$, where $\oplus$ is the sign of the direct sum.

Further, for $k=0,1$ the projection operator $P_{k}(H)$ onto subspace $R_{k}(H)$ is identified with the matrix $P_{k}(H), k=0$, 1 , corresponding to it in a fixed basis in $R^{m}$.

Statement 5: If $k=0,1$, and matrix $P_{k}(A(t, x(t)))$ is projector on $R_{k}(A(t, x(t)))$, then:

1) $P_{0}(A(t, x(t)))+P_{1}(A(t, x(t)))=E,(E$ is unity matrix);

2) projectors $P_{0}(A(t, x(t))), P_{1}(A(t, x(t)))$ are orthogonal;

3) $P_{k}^{2}(A(t, x(t)))=P_{k}(A(t, x(t))) \quad[6]$;

4) $P_{1}(A(t, x(t)))=A(t, x(t)) A^{+}(t, x(t)) \quad$ [6].

The solution of the Cauchy problem (3) is of the form

$$
\begin{aligned}
w_{0}(t)= & \kappa(t, a ; A(\cdot)) x_{a}+\int_{a}^{t} \kappa(t, \tau ; A(\cdot))(g(\tau, x(\tau)) \\
& \left.+\frac{1}{\alpha(h)} A(\tau, x(\tau)) x(\tau)\right) d \tau
\end{aligned}
$$

here $\kappa(t, \tau ; A(\cdot))$ - is a solution of the equation

$$
\frac{\partial}{\partial \tau}(\kappa(t, \tau ; A(\cdot)))=\frac{1}{\alpha(h)} \kappa(t, \tau ; A(\cdot)) A(\tau, x(\tau))
$$

with initial condition $\kappa(t, t ; A(\cdot))=E$.

Integration by parts from $a$ to $t$ on the right-hand side 
of (5), and taking (2) and (6) into account, we obtain

$$
\frac{x(t)-w_{0}(t)}{\alpha(h)}=\frac{1}{\partial(h)} \int_{a}^{t} \kappa(t, \tau ; A(\cdot)) f(\tau, x(\tau) d \tau .
$$

Note a few properties of the functions in the right part of (7). According to statement 2 and point 4 of a statement 5 we have

$$
\begin{gathered}
\left(x(t)-w_{0}(t)\right) / \alpha(h)=1 / \alpha(h) \int_{a}^{t} \kappa(t, \tau ; A(\cdot)) \\
\times P_{1}(A(\tau, x(\tau))) A(\tau, x(\tau)) F(\tau) d \tau,
\end{gathered}
$$

where $F(\tau)=\left(f^{T}(\tau, x(\tau))\right)^{+} v(\tau)$.

Both parts of the previous equality are multiplied on $P_{1}(A(t, x(t)))$ :

$$
\begin{aligned}
& P_{1}(A(t, x(t))) \frac{x(t)-w_{0}(t)}{\alpha(h)}=\frac{1}{\alpha(h)} \int_{a}^{t} \kappa_{1}(t, \tau ; A(\cdot)) \\
& \times P_{1}(A(\tau, x(\tau))) A(\tau, x(\tau)) F(\tau) d \tau,
\end{aligned}
$$

where $\kappa_{1}(t, \tau ; A(\cdot))=P_{1}(A(t, x(t))) \kappa(t, \tau ; A(\cdot))$ is a solution of the differential equation (6) with initial condition $\kappa_{1}(t, t ; A(\cdot))=P_{1}(A(t, x(t)))$.

Statement 6: [2] Suppose that assertions of statement 1 hold and for all $t \in[a, b] 1)$ rank $(f(t, x(t)))$ is equal $r, 2)$ the infima with respect to $t$ of distances between the boundaries of compactumes $Q$ and $X$ and $v_{*}(t)$ and $x(t)$, respectively, are positive. Then there exist positive constants $h_{1}, K_{1}, K_{2}$ such that, for all $t \in[a, b], \tau \in[a, t]$, $h \in\left(0, h_{1}\right]$ the following estimation holds

$$
\left\|\kappa(t, \tau ; A(\cdot)) P_{1}(A(\tau, x(\tau)))\right\| \leqslant K_{1} e^{-\frac{\lambda(t-\tau)}{4 \alpha(h)}}+\alpha(h) K_{2},
$$

here $\lambda$ is positive constant such that, for all $t \in[a, b]$ minimal positive eigenvalue $\lambda_{r}(t)$ of matrix $A(t, x(t))$ satisfies the inequality $\lambda_{r}(t) \geqslant \lambda>0$.

Corollary 1 According to (8) and boundedness $F(t)$ such positive constant $K_{0}$ exists that for all $t \in[a, b]$ inequality $\mid\left(x(t)-w_{0}(t) / \alpha(h) \mid \leqslant K_{0}\right.$ is valid.

Corollary 2 For all $t \in[a, b], \tau \in[a, t], h \in\left(0, h_{1}\right]$ the following inequality holds:

$$
\left\|\kappa_{1}(t, \tau ; A(\cdot)) P_{1}(A(\tau, x(\tau)))\right\| \leqslant K_{1} e^{-\frac{\lambda(t-\tau)}{4 \alpha(h)}}+\alpha(h) K_{2}
$$

Definition. Suppose that $h \in(0, \infty), \Phi h(\cdot):[a, b] \times$ $[a, b] \rightarrow R^{m \times m}, \varphi(\cdot):[a, b] \rightarrow R^{m}$ and $\Phi_{h}(t, t) \varphi(t)=\varphi(t)$. Consider the representation $\int_{a}^{t} \Phi_{h}(t, s) \varphi(s) d s=\varphi(t)$ $+\varepsilon(h)$. Let's name the integral operator on the left-hand side of this equality is operator of reconstruction of the value of $\varphi(t) ; \Phi_{h}(t, s)$ - is its kernel, and $\varepsilon(h)$ error of the reconstruction.
Consider a function

$$
\Phi_{h}^{(1)}(t, \tau)=\frac{\partial}{\partial \tau}\left(\kappa_{1}(t, \tau ; A(\cdot)) P_{1}(A(\tau, x(\tau)))\right) .
$$

Let us show that

$$
\int_{a}^{t} \Phi_{h}^{(1)}(t, \tau) F(\tau) d \tau
$$

is the operator of reconstruction of the value of $F(t)$, and let us estimate the error of the reconstruction. It is not difficult to receive the following results.

Lemma 1: If matrix $H(\cdot):[a, b] \rightarrow R^{m \times m}$, mapping $p(\cdot):[a, b] \rightarrow R^{m}$ satisfies of the Lipschitz condition with constant $L_{p}$ and for all $t \in[a, b]$ the representations $\|H(t)\| \leqslant M_{H},\left\|\int_{a}^{t} H(\tau) d \tau\right\| \leqslant \varepsilon$ are valid, then $\mid \int_{a}^{t} H(\tau)$. $(p(\tau)-p(t)) d \tau \mid \leqslant \varepsilon L_{p}(b-a)$.

Lemma 2: If $t \in[a, b], v(\cdot)$ satisfies of the condition Lipschitz, and the rank of the matrix $f(t, x(t))$ is constant, then for all $t_{1}, t_{2} \in[a, b]$ there is constant $L_{F}=\frac{6}{\lambda} L M_{v}$. $\left(1+M_{g}+M_{f} M_{v}\right)+\frac{L_{v}}{\sqrt{\lambda}}$ so that $\left|F\left(t_{1}\right)-F\left(t_{2}\right)\right| \leqslant L_{F} \mid t_{1}-$ $t_{2} \mid$

The formulated lemmas allow to pass to an estimation the error of the reconstruction operator of the function value:

Lemma 3: Suppose that condition (*) hold; $\delta(h)$, $\frac{\alpha(h)}{\delta(h)}$ tend to zero together with $h, v(t) \equiv 0$ for $t \in[a-$ $\delta(h), a)$ and $k \in \mathbb{N}$. Then there exist positive constants $h_{2}(k), K_{3}, K_{4}$ such that, for $h \in\left(0, h_{2}(k)\right]$ the error of operator of reconstruction of the value of $F(t)$ with kernel $\Phi_{h}^{(1)}(t, \tau)$ satisfies the estimation

$$
\varepsilon(h) \leqslant\left(2 K_{1}+K_{2}\right) L_{F} \delta(h)+K_{3}\left(\frac{4 \alpha(h)}{\lambda \delta(h)}\right)^{k}+\alpha(h) K_{4} .
$$

Proof. Let's put $\Phi_{h}^{(1)}(t, \tau)=\Phi_{h}^{(1)}(t, a)$ when $\tau \in[a-$ $\delta(h), a)$, and define

$$
\begin{gathered}
I_{1}=\left|\int_{a-\delta(h)}^{t-\delta(h)} \Phi_{h}^{(1)}(t, \tau)(F(\tau)-F(t)) d \tau\right|, \\
I_{2}=\left|\int_{t-\delta(h)}^{t} \Phi_{h}^{(1)}(t, \tau)(F(\tau)-F(t)) d \tau\right|, \\
I_{3}=\left|\int_{a-\delta(h)}^{t} \Phi_{h}^{(1)}(t, \tau) F(t) d \tau-F(t)\right| .
\end{gathered}
$$

Let's estimate each of these quantities. According to lemmas 1, 2, and statements 3, 6 for $I_{1}$ are valid: 


$$
\begin{gathered}
I_{1} \leqslant\left(L_{F}(b-a)+|F(t-\delta(h))|+|F(t)|\right) \\
\times\left\|\int_{a-\delta(h)}^{t-\delta(h)} \Phi_{h}^{(1)}(t, \tau) d \tau\right\| \leqslant\left(L_{F}(b-a)+\frac{2}{\sqrt{\lambda}} M_{v}\right) \\
\times \| \kappa_{1}(t, t-\delta(h) ; A(\cdot)) P_{1}(A(t-\delta(h), x(t-\delta(h))) \\
-\kappa_{1}(t, a-\delta(h) ; A(\cdot)) P_{1}(A(a-\delta(h), x(a-\delta(h))) \| \\
\leqslant\left(L_{F}(b-a)+\frac{2 M_{v}}{\sqrt{\lambda}}\right)\left(K_{1} e^{-\frac{\lambda \delta(h)}{4 \alpha(h)}}+K_{1} e^{-\frac{\lambda(t-a+\delta(h))}{4 \alpha(h)}}\right. \\
\left.+2 \alpha(h) K_{2}\right) \leqslant 2\left(K_{1} e^{-\frac{\lambda \delta(h)}{4 \alpha(h)}}+\alpha(h) K_{2}\right) \cdot \\
\left(L_{F}(b-a)+\frac{2}{\sqrt{\lambda}} M_{v}\right) ;
\end{gathered}
$$

for $I_{2}: I_{2} \leqslant L_{F} \delta(h) \int_{t-\delta(h)}^{t}\left\|\Phi_{h}^{(1)}(t, \tau) d \tau\right\| \leqslant\left(2 K_{1}+K_{2}\right)$. $L_{F} \delta(h)$;

for $I_{3}$, according to statements 2 and 6 :

$$
\begin{aligned}
& I_{3} \leqslant \mid\left[\kappa_{1}(t, t ; A(\cdot)) P_{1}(A(t, x(t)))\right. \\
& -\kappa_{1}(t, a-\delta(h) ; A(\cdot)) P_{1}(A(a-\delta(h), x(a-\delta(h))) \\
& \left.-P_{1}(A(t, x(t)))\right] F(t) \mid \leqslant\left(K_{1} e^{-\frac{\lambda \delta(h)}{4 \alpha(h)}}+2 \alpha(h) K_{2}\right) \frac{M_{v}}{\sqrt{\lambda}}
\end{aligned}
$$

The error of the operator of reconstruction defined by (10) satisfies the inequality

$$
\begin{aligned}
& \varepsilon(h)=\left|\int_{a}^{t} \Phi_{h}^{(1)}(t, \tau) F(\tau) d \tau-F(t)\right| \\
& \leqslant\left|\int_{a-\delta(h)}^{t-\delta(h)} \Phi_{h}^{(1)}(t, \tau)(F(\tau)-F(t)) d \tau\right| \\
& +\left|\int_{t-\delta(h)}^{t} \Phi_{h}^{(1)}(t, \tau)(F(\tau)-F(t)) d \tau\right| \\
& +\left|\int_{a-\delta(h)}^{t} \Phi_{h}^{(1)}(t, \tau) F(\tau) d \tau-F(t)\right| .
\end{aligned}
$$

The last, taking into account the estimations for $I_{j}$ at $K_{3}=K_{1}\left(2 L_{F}(b-a)+\frac{5 M_{v}}{\sqrt{\lambda}}\right), \quad K_{4}=2 K_{2}\left(L_{F}(b-a)+\frac{3 M_{v}}{\sqrt{\lambda}}\right)$, implies relation $\varepsilon(h) \leqslant\left(2 K_{1}+K_{2}\right) L_{F} \delta(h)+K_{3} e^{-\frac{\lambda \delta(h)}{4 \alpha(h)}}$ $+\alpha(h) K_{4}$.

Note that, for any $k \in \mathbb{N}$ we can indicate such $h_{2}(k)$ $>0$ that, for all $h \in\left(0, h_{2}(k)\right]$ inequality $e^{-\frac{\lambda \delta(h)}{4 \alpha(h)}} \leqslant$ $\left(\frac{4 \alpha(h)}{\lambda \delta(h)}\right)^{k}$ is valid.

This fact implies the assertion of the lemma.
Let's pass from the integral on the right-hand side of (9) to the operator of reconstruction of value $F(t)$ with kernel $\Phi_{h}^{(1)}(t, \tau), t \in[a, b], \tau \in[a, t]$.

According to (6), $\kappa_{1}(t, \tau ; A(\cdot)) P_{1}(A(\tau, x(\tau)))$ is a solution the problem of Cauchy

$$
\begin{gathered}
\frac{\partial}{\partial \tau}\left(\kappa_{1}(t, \tau ; A(\cdot)) P_{1}(A(\tau, x(\tau)))\right)=\frac{\kappa_{1}(t, \tau ; A(\cdot))}{\alpha(h)} \\
\times P_{1}(A(\tau, x(\tau))) A(\tau, x(\tau))+\kappa_{1}(t, \tau ; A(\cdot)) \\
\times \frac{d}{d \tau}\left(P_{1}(A(\tau, x(\tau))),\right. \\
\kappa_{1}(t, t ; A(\cdot)) P_{1}(A(t, x(t)))=P_{1}(A(t, x(t))),
\end{gathered}
$$

which implies that (9) takes the form:

$$
\begin{gathered}
P_{1}(A(t, x(t))) \frac{x(t)-w_{0}(t)}{\alpha(h)}=\int_{a}^{t} \Phi_{h}^{(1)}(t, \tau) F(\tau) d \tau \\
-\int_{a}^{t} \kappa_{1}(t, \tau ; A(\cdot)) \frac{d}{d \tau}\left(P_{1}(A(\tau, x(\tau)))\right) F(\tau) d \tau
\end{gathered}
$$

In [2] the following result has been received:

Lemma 4: If conditions of statement 6 are satisfied, then there are such positive constant $K_{4}$ and $h_{3}$ that for all $t \in[a, b], \quad \tau \in[a, t], \quad h \in\left(0, h_{3}\right]$

$$
\left\|\kappa_{1}(t, \tau ; A(\cdot)) P_{0}(A(\tau, x(\tau)))\right\| \leqslant \alpha(h) K_{4}
$$

According to the approach offered in [2], it is not difficult to receive the following result.

Lemma 5: Suppose that the assumptions of lemma 3 hold. Then there exist positive constants $K_{5}, K_{6}, K_{7}$ such that, for all $t \in[a, b]$ the following estimate is valid.

$$
\left|v_{0}(t)-v_{*}(t)\right| \leqslant K_{7} \delta(h)+K_{8}\left(\frac{4 \alpha(h)}{\lambda \delta(h)}\right)^{k}+K_{9} \alpha(h) .
$$

\section{Estimate of the Norm of Difference between $v_{0}(\cdot)$ and $v_{h}(\cdot)$}

To derive this estimate, we need, first, to estimate $\left|w_{h}(t)-w_{0}(t)\right|$. Note that the rule

$$
\begin{aligned}
w_{h}(t)= & w_{h}\left(t_{i}\right)+\left(g\left(t_{i}, x\left(t_{i}\right)\right)+f\left(t_{i}, x\left(t_{i}\right)\right) v_{i}\right) \\
& \times\left(t-t_{i}\right), \quad t \in\left[t_{i}, t_{i+1}\right), \quad v_{0}=v_{\sigma}(a)
\end{aligned}
$$

can be regarded as the implementation of the Euler method for solving problem (3) with an inexact calculated righthand side. In view of the specific character of our equation, we cannot use familiar results. For obtaining of a required estimation we will adhere to the approach offered in [2]. For simplicity we assume in what follows that $\frac{b-a}{\Delta(h)} \in \mathbb{N}$. 
Consider the Euler method for the differential equation (3) with exactly calculated right-hand side: for $t \in\left[t_{i}\right.$,

$$
\begin{aligned}
& \left.t_{i+1}\right) \\
& w_{e}(t)=w_{e}\left(t_{i}\right)+\left(g\left(t_{i}, x\left(t_{i}\right)\right)+\frac{1}{\alpha(h)} A\left(t_{i}, x\left(t_{i}\right)\right)\right. \\
& \left.\quad \times\left(x\left(t_{i}\right)-w_{e}\left(t_{i}\right)\right)\right)\left(t-t_{i}\right), \quad w_{e}(a)=w_{0}(a) .
\end{aligned}
$$

In [2] the following result has been received:

Lemma 6: Let condition (*) hold. Then there exist positive constants $h_{4}, K_{10}$ such that for all $h \in\left(0, h_{4}\right]$ and $t \in[a, b]$ the following estimate holds:

$$
\left|w_{0}(t)-w_{e}(t)\right| \leqslant \frac{\Delta(h)}{\alpha(h)} K_{10}
$$

Lemma 7: Let condition (*) hold. Then there exist positive constants $h_{5}, K_{12}, K_{13}$ such that for all $h \in\left(0, h_{5}\right]$ and $t \in[a, b]$ the following estimate holds:

$$
\left|w_{h}(t)-w_{e}(t)\right| \leqslant \frac{h}{\alpha(h)} K_{12}+\sigma(h) K_{13} .
$$

Proof. According to (11) and (12), the following relation holds

$$
\begin{gathered}
w_{h}\left(t_{i+1}\right)-w_{e}\left(t_{i+1}\right)=\left(E-A\left(t_{i}, x_{h}\left(t_{i}\right)\right) \frac{\Delta(h)}{\alpha(h)}\right. \\
\times\left(w_{h}\left(t_{i}\right)-w_{e}\left(t_{i}\right)\right)+A\left(t_{i}, x_{h}\left(t_{i}\right)\right) \frac{x_{h}\left(t_{i}\right)-x\left(t_{i}\right)}{\alpha(h)} \\
\times \Delta(h)+\left(A\left(t_{i}, x_{h}\left(t_{i}\right)\right)-A\left(t_{i}, x\left(t_{i}\right)\right)\right) \\
\times\left(\frac{x\left(t_{i}\right)-w_{0}\left(t_{i}\right)}{\alpha(h)}+\frac{w_{0}\left(t_{i}\right)-w_{e}\left(t_{i}\right)}{\alpha(h)}\right) \Delta(h) \\
\quad+\left(g\left(t_{i}, x_{h}\left(t_{i}\right)\right)-g\left(t_{i}, x\left(t_{i}\right)\right)\right) \Delta(h),
\end{gathered}
$$

Taking into account $(*)$, corollary 1 from statement 6 and lemma 6 the following estimations hold:

$$
\begin{gathered}
\left|x_{h}(t)-x\left(t_{i}\right)\right| \leqslant h ;\left|\frac{x\left(t_{i}\right)-w_{0}\left(t_{i}\right)}{\alpha(h)}\right| \leqslant K_{0} ; \\
|| A\left(t_{i}, x_{h}\left(t_{i}\right)\right)-A\left(t_{i}, x\left(t_{i}\right)\right) \mid \leqslant 2 L M_{f} h ; \\
\left|w_{0}\left(t_{i}\right)-w_{e}\left(t_{i}\right)\right| \leqslant \frac{\Delta(h)}{\alpha(h)} K_{11} ; \\
\left|g\left(t_{i}, x_{h}\left(t_{i}\right)\right)-g\left(t_{i}, x\left(t_{i}\right)\right)\right| \leqslant h\left(L+L M_{v}\right)+\sigma M_{f},
\end{gathered}
$$

hence there exist positive constant $h_{5}, K_{11}=M_{f}^{2}+2 L M_{f} K_{0}$ $+4 L M_{f} M_{10}+L\left(1+M_{v}\right)$ such that, for all $h \in\left(0, h_{5}\right]$

$$
\begin{gathered}
\left|w_{h}\left(t_{i+1}\right)-w_{e}\left(t_{i+1}\right)\right| \leqslant\left|w_{h}\left(t_{i}\right)-w_{e}\left(t_{i}\right)\right| \\
+\frac{h \Delta(h)}{\alpha(h)} K_{11}+\sigma(h) \Delta(h) M_{f}
\end{gathered}
$$

Since, in this case, $\left|w_{h}\left(t_{0}\right)-w_{e}\left(t_{0}\right)\right| \leqslant h$, it follows from the (13) that, for any $i=1, n$ :

$$
\begin{gathered}
\left|w_{h}\left(t_{i}\right)-w_{e}\left(t_{i}\right)\right| \leqslant h+n\left(\frac{h \Delta(h)}{\alpha(h)} K_{11}+\sigma(h) \Delta(h) M_{f}\right) \\
=h+\frac{b-a}{\Delta(h)}\left(\frac{\Delta(h) h}{\alpha(h)} K_{11}+\sigma(h) \Delta(h) M_{f}\right) \\
\leqslant \frac{h}{\alpha(h)} K_{12}+\sigma(h) K_{13},
\end{gathered}
$$

$K_{12}=(b-a) K_{12}+1, K_{13}=(b-a) M_{f}$. The lemma is proved.

Using lemmas 6, 7, we obtain the following result.

Lemma 8: Let the assumption of lemma 6 hold, $\Delta(h)$ $=h$. Then there exist positive constant $K_{14}, K_{15}$ such that, for all $t \in[a, b]$, the following estimate hold:

$$
\left|w_{h}(t)-w_{0}(t)\right| \leqslant \frac{h}{\alpha(h)} K_{14}+\sigma(h) K_{15} .
$$

Note that the difference between $v_{0}(t)$ and $v_{h}(t)$ for $t \in\left[t_{i}, t_{i+1}\right)$ can be expressed as:

$$
\begin{aligned}
v_{0}(t) & -v_{h}(t)=f^{T}(t, x(t)) \frac{x(t)-x_{h}\left(t_{i}\right)}{\alpha(h)} \\
& +f^{T}(t, x(t)) \frac{w_{h}\left(t_{i}\right)-w_{h}(t)}{\alpha(h)} \\
& +f^{T}(t, x(t)) \frac{w_{h}(t)-w_{0}(t)}{\alpha(h)} \\
+ & \left(f^{T}(t, x(t))-f^{T}\left(t_{i}, x_{h}\left(t_{i}\right)\right)\right) \frac{x_{h}\left(t_{i}\right)-w_{h}\left(t_{i}\right)}{\alpha(h)}
\end{aligned}
$$

In view of (14) and lemma8 the following result hold:

Lemma 9: Suppose that the assumptions of lemma 8 hold, quantities $\frac{h}{\alpha^{2}}, \frac{\sigma(h)}{\alpha(h)}$ are bounded. Then there exist positive constants $K_{v}, h_{6}$ such that $\left|v_{h}(t)\right| \leqslant K_{v}$ for all $h \in\left(0, h_{6}\right]$ and $t \in[a, b]$.

According to approach, considered in [2], we can obtain the next result. It is required to obtain a sharper estimate.

Lemma 10: Suppose that assumptions of lemma 9 hold. Then there exist constants $K_{16}, K_{17}$ such that for all $t \in[a, b]$ the following inequality holds:

$$
\left|w_{0}(t)-w_{h}(t)\right| \leqslant h K_{16}+\alpha(h) \sigma(h) K_{17} .
$$

Let's now refine the norm of the difference between $v_{0}(\cdot)$ and $v_{h}(\cdot)$. From the (14), the condition $(*)$, lemmas 5 and 10 the next result hold.

Lemma 11: Suppose that the assumptions the lemma 8 hold, then there exist constants $K_{18}, K_{19}>0$ that for all $t \in[a, b]$ the following inequality holds 


$$
\left|v_{0}(t)-v_{h}(t)\right| \leqslant \frac{h}{\alpha(h)} K_{18}+\sigma(h) K_{19} .
$$

\section{The Upper Estimation, the Asymptotic Order of Accuracy}

It is known that there exist constant $K_{20}>0$ such that the lower estimation of accuracy $D_{h}$ in $C[a, b]$ is of the form

$$
v_{1}(h) \geqslant K_{20} \sqrt{h}
$$

In view of lemmas 5,11 , the following estimate hold:

Theorem 1: (upper bound for the accuracy). Let condition $(*)$ hold and $\delta_{1}(h)=\frac{\lambda}{4} \delta(h)$. Then the upper bound for the accuracy in $C[a, b]$ is of the form:

$$
\begin{aligned}
v_{2}(h) \leqslant & \frac{4 K_{7}}{\lambda} \delta_{1}(h)+K_{8}\left(\frac{\alpha(h)}{\delta_{1}(h)}\right)^{k}+\alpha(h) K_{9} \\
& +\frac{h}{\alpha(h)} K_{18}+\sigma(h) K_{19} .
\end{aligned}
$$

Remark 1: Optimal on $h$ the order of upper estimation of accuracy may be realized, at choice $\delta_{1}(h)=h^{\frac{k}{2 k+1}}$, $\alpha(h)=h^{\frac{k+1}{2 k+1}}, \sigma(h)=\alpha(h)$, hence $\gamma_{0}=\frac{1}{2}$.

Remark 2: In our case the unknowing impact $u_{*}(\cdot)$ can be defined as $u_{*}(\cdot)=v_{*}(\cdot)+P_{1}(A(t, x(t))) u(a)$.

\section{References}

[1] Y. S. Osipov and A. V. Kryazhimskii, "Inverse Problems for Ordinary Differential Equations: Dynamical Solutions," Gordon and Breach Science Publishers, London, 1995.

[2] A. Y. Vdovin and S. S. Rubleva, "On the Guaranteed Accuracy of a Dynamical Recovery Procedure for Controls with Bounded Variation in Systems Depending Linearly on the Control," Mathematical Notes, Vol. 87, No. 3, 2010, pp. 316-335.

[3] A. Y. Vdovin, A. V. Kim and S. S. Rubleva, "On Asymptotic Accuracy in L1 of One Dynamical Algorithm for Reconstructing a Disturbance," Proceedings of the Steklov Institute of Mathematics, Vol. 255, 2006, pp. 216-224.

[4] Y. S. Osipov, F. P. Vasilyev and M. M. Potapov, "Bases of the Method Dynamic Regulation," in Russian, Moscow State University Press, Moscow, 1999.

[5] V. V. Voevodin and Y. A. Kuznetzov, "Matrixes and Calculations," in Russian, Publishing House "Nauka", Moscow, 1984.

[6] A. Albert, "Regression and the Moor-Penrose Pseudoinverse," Academic Press, New York, 1972.

[7] P. A. Wedin, "Pertubation Theory for Pseudoinverces," BIT Numerical Mathematics, Vol. 13, No. 2, 1973, pp. 217-232. 